MODELING, IDENTIFICATION AND CONTROL, 1996, vOL. 17, NO. 2, 87-96

doi:10.4173/mic.1996.22

\title{
Wave disturbance filtering in dynamic positioning systems
}

\author{
TOR STEINAR SCHEI $\dagger$ \\ Keywords: Marine systems; ship control; navigation; filtering; state estimation \\ Three different approaches to wave disturbance filtering in dynamic positioning \\ systems are studied in this paper. It is shown that a conventional design based on \\ notch filters leads to approximately the same achievable performance of the total \\ control system as can be achieved with an observer based design. It is also shown \\ that a proposed passivity based design leads to a conventional filter with \\ PD-controller. However, there is a relation between the parameters in the filter and \\ the PD-controller, which ensures the passivity properties of the control system.
}

\section{Introduction}

This paper discusses various wave filtering techniques used in dynamic positioning (DP) systems for ships and other surface vessels. The purpose of such wave filters is twofold; to reduce fuel consumption and wear on the propulsion equipment (e.g. propellers and thruster actuators). Several approaches for the design of wave filters have been proposed in the literature and implemented in commercial control systems.

The environmental forces acting on a surface vessel is generally complicated. Sea waves consist of a large number of oscillatory components with various directions of propagation, different amplitudes and phases. The resulting wave spectrum usually has a peak value in the interval $0.3<\omega_{0}<1.3(\mathrm{rad} / \mathrm{s})$, where $\omega_{0}$ is the dominating frequency component. Waves in this frequency range generate large oscillatory forces and moments on the vessel, and the wave induced motion is difficult to handle with the control system; $\omega_{0}$ is often just above the control bandwidth of most vessels, but still low enough to induce a significant vessel motion. Further, these disturbances will be inside the bandwidth of the servos and actuators of the vessel. In addition to the first order wave forces there are also more slowly varying disturbances caused by wind, current and wave drift forces (second order wave forces). These disturbances can usually be suppressed by the control system. The purpose of the wave disturbance filter is then to separate the oscillatory wave induced motion from the motion caused by the more slowly varying disturbances and by the vessel actuators. Thruster modulation is then reduced by implementing feedback from the low frequency vessel motion.

\section{Filtering methods}

Dynamic positioning (DP) systems have been commercially available since the early 1960s. The early DP systems were designed using conventional PID-controllers for control of the motion in surge, sway and yaw, under the assumption that interactions between the three loops were negligible (Sargent and Cowgill 1976; Morgan 1978). A notch filter or other conventional filter is introduced in each control loop in order to

Received 20 August 1995

† SINTEF Automatic Control, 7034 Trondheim, Norway. Fax: + 477359 43 99, Phone: +4773594375 .

Presented at the 3rd IFAC Workshop on Control Applications in Marine Systems, Trondheim, Norway, May 1995 (CAMS '95). 
reduce thruster modulation due to first-order wave disturbances. Since 1975 more advanced techniques, such as Kalman filtering and optimal control have been applied to DP systems (Balchen et al. 1980; Grimble and Patton 1980; Sælid et al. 1983). In these approaches the vessel is modeled as a combination of a low-frequency (LF) and a high frequency (HF) model. the LF model represents the motion caused by the propulsion system, wind current and second order wave forces while the HF model represents the first-order wave induced motion. Recently, an approach based on a passive controller structure has also been proposed (Johannesen and Egeland 1993).

These three different approaches are compared below. The properties of conventional filters and the impact of such filters on ordinary feedback loops are quite easy to analyze by conventional methods. A major disadvantage with conventional filtering techniques such as notch, low pass and band pass filters in feedback systems is the negative phase lags added to the phase of the feedback loops. The introduction of observer (e.g. Kalman filter) based filtering techniques was partly motivated by this deficiency of conventional filtering. There seems to be an underlying assumption in several sources that observer based filtering techniques introduce less negative phase lags into the feedback loops than conventional filter designs. This assumption is addressed in the following.

\section{Model of vessel motion}

A simple linear model will be used for the analysis in this paper. The model is linearized at zero speed and the current is assumed to be zero. The interactions between the vessel motion in surge, sway and yaw are then zero. Further, linear drag is neglected and the vessel motion can then be modeled as a double integrator in each axis of motion. The low frequency vessel motion in the surge direction is modeled as:

$$
\begin{aligned}
& \dot{x}_{L_{L}}=u_{L_{L}} \\
& \dot{u}_{L}=\frac{1}{m} F_{T}+\eta_{L}
\end{aligned}
$$

where $u_{L}$ and $x_{L}$ are the low frequency components of the vessel speed and position in the surge direction, $F_{T}$ is the thrust force in the surge direction and $m$ is the mass of the vessel including added mass in the surge direction. $\eta_{L}$ represents disturbances other than first order wave forces. Similar models are established for the vessel motion in the sway and yaw directions.

(1) is certainly too simple for design and analysis of a high performance DP system; interactions between the three axes of motion cannot be neglected when the vessel has nonzero speed relative to the water, and nonlinear drag is certainly significant. Anyway, (1) is assumed to be sufficiently accurate for analysis of wave filtering techniques. A more accurate model of the vessel motion can be found in Sælid et al. (1983), more general model formulations are found in Fossen (1994).

The first order wave induced motion is modeled as a damped oscillator in accordance with Sælid et al. (1983):

$$
\begin{aligned}
& \dot{x}_{H}=u_{H}+c_{1} \eta_{H} \\
& \dot{u}_{H}=-2 \zeta \omega_{0} u_{H}-\omega_{0}^{2} x_{H}+c_{2} \eta_{H}
\end{aligned}
$$


where $x_{H}$ is the wave induced motion of the vessel and $\eta_{H}$ is assumed to be white noise with variance equal one, $\operatorname{cov}\left(\eta_{H}\right)=1 \cdot 0$.

The low-frequency (LF) and the high-frequency (HF) model transfer functions are:

$$
\begin{aligned}
& x_{L}(s)=h_{L}(s) F_{T}(s)+\frac{1}{s^{2}} \eta_{L}(s), \quad h_{L}(s)=\frac{1}{m s^{2}} \\
& x_{H}(s)=\frac{c_{1} s}{s^{2}+2 \zeta \omega_{0} s+\omega_{0}^{2}} \eta_{H}(s)=h_{H}(s) \eta_{H}(s)
\end{aligned}
$$

The following relation is used: $c_{2}+2 \zeta c_{1}=0$, since the spectrum of the high-frequency motion is zero at zero frequency. The spectrum $\Phi_{x_{H} x_{H}}(j \omega)=\left|h_{H}(j \omega)\right|^{2}$ of the high-frequency induced motion has been verified from real measurement data with good agreement (Sælid 1983). Higher order models for the wave induced vessel motion have also been proposed, see for example Grimble and Patton (1980). Fossen (1994) gives an overview of different models for sea spectrum approximations.

\section{Conventional wave filter design}

A notch filter has the following transfer function:

$$
f_{n}(s)=\frac{s^{2}+2 \zeta_{n} \omega_{n} s+\omega_{n}^{2}}{s^{2}+2 \zeta_{d} \omega_{n} s+\omega_{n}^{2}}
$$

where $\zeta_{\mathrm{n}} / \zeta_{\mathrm{d}}<1$. Some important characteristics of this filter are:

- $f_{n}(j \omega) \approx 1$ for $\omega \ll \omega_{n}$ or $\omega \gg \omega_{n}$.

- $f_{n}\left(j \omega_{n}\right)=\zeta_{n} / \zeta_{d}$.

- $\left|\angle f_{n}(j \omega)\right|<90^{\circ}$ for all $\omega$.

The width of the notch is mainly determined by $\zeta_{n}$, and the depth is determined by $\zeta_{n} / \zeta_{d}$. Some realistic values on the filter parameters can be: $\omega_{n}=0 \cdot 5, \zeta_{n}=0 \cdot 1$ and $\zeta_{d}=1 \cdot 0$.

It is assumed that the vessel is controlled by a PD-controller:

$$
c_{P D}(s)=K_{P}\left(1+T_{D} s\right)
$$

and the total loop transfer function for the vessel, the notch filter and the PD-controller is then (the return ratio):

$$
g(s)=h_{L}(s) f_{n}(s) c_{P D}(s)
$$

\section{Observer based wave filter design}

Observer based wave filter designs have been proposed by Balchen et al. (1980) and by Grimble and Patton (1980). These filters are first studied in terms of general linear models for the low-frequency vessel motion and for the high-frequency wave induced motion. Hence, the models (1) and (2) are generalized. The low-frequency model is stated as:

$$
\begin{aligned}
& \dot{\boldsymbol{x}}_{L}=A_{L} \boldsymbol{x}_{L}+B_{L} \boldsymbol{u}+\boldsymbol{\eta}_{L} \\
& \boldsymbol{y}_{L}=C_{L} \boldsymbol{x}_{L}
\end{aligned}
$$

where $\boldsymbol{u}$ is the vector of control inputs, $\boldsymbol{x}_{L}$ is the state vector of the LF-model, and $\boldsymbol{y}_{L}$ is the low frequency part of the total measurement vector. $\boldsymbol{\eta}_{L}$ is the vector of disturbances other than first order wave disturbances. 
The high frequency model is stated as:

$$
\begin{aligned}
& \dot{\boldsymbol{x}}_{H}=A_{H} \boldsymbol{x}_{H}+\boldsymbol{\eta}_{H} \\
& \boldsymbol{y}_{H}=C_{H} \boldsymbol{x}_{H}
\end{aligned}
$$

where $x_{H}$ is the state vector of the HF-model and $y_{L}$ is the high frequency part of the total measurement vector. $\eta_{H}$ is a vector of disturbances. The total measurement vector is now:

$$
y=y_{L}+y_{H}+w
$$

where $\boldsymbol{w}$ is the measurement noise. An observer based on (7)-(9) can be formulated as follows in the frequency domain:

$$
\begin{aligned}
\hat{y}_{L}(s) & =C_{L}\left(s I-A_{L}\right)^{-1} B_{L} \boldsymbol{u}(s)+C_{L}\left(s I-A_{L}\right)^{-1} K_{L}(y(s)-\hat{y}(s)) \\
\hat{y}_{H}(s) & =C_{H}\left(s I-A_{H}\right)^{-1} K_{H}(y(s)-\hat{y}(s)) \\
\hat{y}(s) & =\hat{y}_{L}(s)+\hat{y}_{H}(s)
\end{aligned}
$$

where $\hat{\boldsymbol{y}}_{L}$ and $\hat{\boldsymbol{y}}_{H}$ are estimates of $\boldsymbol{y}_{L}$ and $\boldsymbol{y}_{H}$, and $\mathrm{K}_{L}, \mathrm{~K}_{H}$ are the observer gain updating matrices for the LF and the HF models. The observer has the same structure as a stationary Kalman filter, if it is assumed that the sequences $\eta_{L}(t), \eta_{H}(t)$ and $\mathbf{w}(t)$ are independent white noise processes. The following transfer function matrices are defined:

$$
\begin{aligned}
& H_{L}(s)=C_{L}\left(s I-A_{L}\right)^{-1} B_{L} \\
& G_{L}(s)=C_{L}\left(s I-A_{L}\right)^{-1} K_{L} \\
& G_{H}(s)=C_{H}\left(s I-A_{H}\right)^{-1} K_{H}
\end{aligned}
$$

A block diagram for this filter is shown in Fig. 1. $v(s)$ is the accumulated effect of all disturbances on the measurement vector $\boldsymbol{y}(s)$.

In the following it is assumed that the vessel model $\mathrm{H}_{L}(s)$ is perfect. The low-frequency estimate can then be expressed as:

$$
\hat{\boldsymbol{y}}_{L}(s)=H_{L}(s) \boldsymbol{u}(s)+M_{F}(s) \boldsymbol{v}(s)
$$

where

$$
\begin{aligned}
M_{F}(s) & =\left[I+G_{F}(s)\right]^{-1} G_{F}(s) \\
G_{F}(s) & =G_{F}(s)\left[I+G_{H}(s)\right]^{-1}
\end{aligned}
$$

$F_{H}(s)=\left[I+G_{H}(s)\right]^{-1}$ is a generalized notch filter which extracts the high-frequency disturbances from the outer loop in Fig. 1. $G_{L}(s)$ can be factored as $G_{L}(s)=H_{L}(s) K_{L}(s)$,

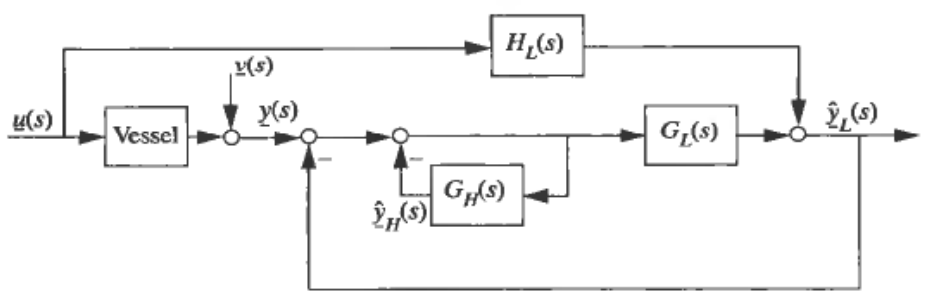

Figure 1. Block diagram for the observer based filter (10), (11). 
if it is assumed that $H_{L}(s)$ is a square transfer function matrix with no right half plane transmission zeros. The transfer function matrix for the outer loop (the return ratio) is then:

$$
G_{F}(s)=H_{L}(s) K_{L}(s) F_{H}(s)
$$

The design of the observer based filter resembles the design of the notch filter and the PD-controller in (6).

It is now assumed that the total control system for the vessel in Fig. 1 is formed by including a high gain state feedback from the estimated state $\hat{\boldsymbol{x}}_{L}(s)$ to the control input $\boldsymbol{u}(s)$. A possible design method is the LQG/LTR approach proposed by Stein and Athans (1987). Pole placement can also be used. It is assumed that the state feedback gain is sufficiently high such that $\hat{y}_{L}(s) \approx 0$, where it is also assumed that the reference is $\boldsymbol{y}_{\text {Ref }}=0$. The control input is then

$$
u(s) \approx-H_{L}^{-1}(s) M_{F}(s) y(s)
$$

and the deviation from the reference is

$$
\boldsymbol{y}(s) \approx\left(I-H_{L}(s) H_{L}^{-1}(s) M_{F}(s)\right) y(s)=\left[I+G_{F}(s)\right]^{-1} \boldsymbol{y}(s)=N_{F}(s) v(s)
$$

where $N_{F}(s)$ is the output sensitivity for the outer observer loop in Fig. 1.

The simple model in Section 3 is considered in the following. An observer based on this model can be expressed as:

$$
\begin{aligned}
\dot{\hat{x}}_{L} & =\hat{u}_{L}+k_{1} \varepsilon \\
\dot{\hat{u}}_{L} & =\frac{1}{m} F_{T}+k_{2} \varepsilon \\
\dot{\hat{x}}_{H} & =\hat{u}_{H}+k_{3} \varepsilon \\
\dot{\hat{u}}_{H} & =-2 \zeta \omega_{0} \hat{u}_{H}-\omega_{0}^{2} \hat{x}_{H}+k_{4} \varepsilon \\
\varepsilon & =y-\hat{x}_{L}-\hat{x}_{H}
\end{aligned}
$$

The filter transfer functions are:

where

$$
\hat{x}_{L}(s)=h_{L}(s) F_{T}(s)+h_{L}(s) k_{L}(s) \varepsilon(s)
$$

$$
k_{L}(s)=m\left(k_{1} s+k_{2}\right)
$$

and

where

$$
\hat{x}_{H}(s)=h_{H}(s) k_{H}(s) \varepsilon(s)=g_{H}(s) \varepsilon(s)
$$

$$
k_{H}(s)=\frac{1}{c_{1} s}\left(k_{3} s+\left(k_{4}+2 \zeta \omega_{0} k_{3}\right)\right)
$$

According to Sælid et al. $(1983): k_{4}+2 \zeta \omega_{0} k_{3}=0$ for the stationary Kalman filter. This means that $g_{H}(s)$ has a zero in the origin. This is only true if the Kalman filter gains are determined with $\eta_{L}=0$ in (1), which results in no updating of the low-frequency model. 
The 'generalized notch filter' is now:

$$
f_{H}(s)=\frac{1}{1+g_{H}(s)}=\frac{s^{2}+2 \zeta \omega_{0} s+\omega_{0}^{2}}{s^{2}+\left(2 \zeta \omega_{0}+k_{3}\right) s+\omega_{0}^{2}+\left(k_{4}+2 \zeta \omega_{0} k_{3}\right)}
$$

and it is clear that $f_{H}(s)$ has the same structure as the notch filter in (4), if $k_{4}+2 \zeta \omega_{0} k_{3}=0$.

The transfer function for the outer loop in Fig. 1 is:

$$
g_{F}(s)=h_{L}(s) k_{L}(s) f_{H}(s)
$$

where $k_{L}(s)$ has a PD-structure. Hence, with $k_{4}+2 \zeta \omega_{0} k_{3}=0$ it is seen that the observer loop for $\hat{y}_{L}(s)$ in fig. 1 has the same structure as the loop transfer function (return ratio) for the conventional notch filter with PD-controller in (6).

\section{Passivity based design}

The passive controller structure proposed by Johannesen and Egeland (1993) is based on the fact that the mapping from thruster force and torque to vessel velocity and angular velocity is passive. A passive controller will then guarantee robust stability of the total nonlinear system in presence of parameter variations and sector nonlinearities.

The proposed controller with wave filter is linear and diagonal, with equal structures in surge, sway and yaw. The transfer functions in surge are expressed in equations (26), (29) and (34) in Johannesen and Egeland (1993):

$$
\begin{aligned}
x_{L}(s) & =\frac{m_{H} s^{2}+K_{D 2} s+K_{P 2}}{m_{H} s^{2}+\left(K_{D 1}+K_{D 2}\right) s+K_{P 1}+K_{P 2}} y(s) \\
F_{T}(s) & =-\left(K_{P 1}+K_{D 1} s\right) x_{L}(s)
\end{aligned}
$$

Hence, it is seen that, with the exception of $K_{P 1}$ in (23), the filter and controller are identical to the conventional notch filter with PD-controller in Section 4. There is, however, a relation between the parameters of the filter in (23) and the PD-controller in (24), which ensures the passivity properties of the control system.

\section{Numerical example}

In this section the performance of an observer based wave filter will be compared with the performance of a conventional filter. It is assumed that (1) is a correct model for the vessel.

The observer gain parameters in (16) and (17) are the stationary Kalman filter parameters, determined under the assumption that the disturbances $\boldsymbol{\eta}_{L}(\mathrm{t})$ and $\boldsymbol{\eta}_{H}(\mathrm{t})$ in (1) and (2) are independent white noise processes, and that the measurement $y(t)$ in (18) is influenced by white additive measurement noise, $w(t)$. the following parameter values are used:

$$
\begin{aligned}
m & =5.0 \times 10^{6} \mathrm{~kg}, \\
\omega_{0} & =0.4 \mathrm{rad} / \mathrm{s}, \\
\zeta & =0.25, \\
c_{1} & =0.4, \\
c_{2} & =-0.08, \\
\operatorname{cov}\left(\eta_{L}(t)\right) & =1.0 \times 10^{-6} \mathrm{~m} / \mathrm{s}^{2}, \\
\operatorname{cov}\left(\eta_{H}(t)\right) & =1.0 \mathrm{~m} / \mathrm{s}^{2}, \\
\operatorname{cov}(w(t)) & =0.1 \mathrm{~m}^{2} .
\end{aligned}
$$


The variance of $\eta_{L}$ is used as a tuning parameter, and the value is determined such that the crossover frequency for the outer filter loop in Fig. 1 is approximately $0.07 \mathrm{rad} / \mathrm{s}$. The variance of the measurement noise is typically $0.1 \mathrm{~m}^{2}$ for acoustic measurement systems. The variance of the continuous measurement above is a reasonable approximation to discrete measurements with a sampling interval of $1.0 \mathrm{~s}$. The other parameters above are from Sælid et al. (1983).

The stationary Kalman filter gain parameters are determined to be:

$$
\begin{aligned}
& k_{1}=9.72 \times 10^{-2}, \\
& k_{2}=3.16 \times 10^{-3}, \\
& k_{3}=1.06, \\
& k_{4}=-0.133 .
\end{aligned}
$$

The feedback gains from $\hat{x}_{L}$ and $\hat{a}_{L}$ are determined such that the two eigenvalues for the feedback system are $\lambda_{1,2}=0 \cdot 1$, hence, the bandwidth of the feedback loop is just above the bandwidth of the estimator loop.

$$
F_{T}=-g_{1} \hat{x}_{L}-g_{2} \hat{u}_{L}
$$

where

$$
\begin{aligned}
& g_{1}=m \lambda_{1,2}^{2}=5.0 \times 10^{4} \\
& g_{2}=2 m \lambda_{1,2}=1.0 \times 10^{6}
\end{aligned}
$$

A higher value of $\lambda_{1,2}$ would result in better recovery of the return ratio for the observer (Stein and Athans, 1987), but with increased high frequency noise amplification. The choice of bandwidth for the state feedback loop is a compromise between low peak sensitivity and low high frequency noise and disturbance amplification for the control system.

The conventional notch filter with PD controller is tuned such that the performance resembles the performance of the observer based filter. The parameters of the notch filter in (4) are set to:

$$
\begin{aligned}
\omega_{n} & =0.4 \mathrm{rad} / \mathrm{s}, \\
\zeta_{n} & =0.25, \\
\zeta_{d} & =\left(2 \zeta \omega_{0}+k_{3}\right) /\left(2 \omega_{n}\right)=1.58 .
\end{aligned}
$$

The PD-parameters are set to:

$$
\begin{aligned}
& K_{p}=2.4 \times 10^{3}, \\
& T_{d}=120 \mathrm{~s} .
\end{aligned}
$$

In order to reduce high frequency noise sensitivity, the measurement is filtered through a low-pass filter:

$$
f_{l}(s)=\frac{1}{\left(1+s / \omega_{f}\right)^{2}}, \quad \omega_{f}=0 \cdot 2 \mathrm{rad} / \mathrm{s}
$$

The results of the comparison between the two control structures with wave filters are shown in Figs 2-5. There are solid lines for the observer and state feedback system and broken lines for the conventional system. 

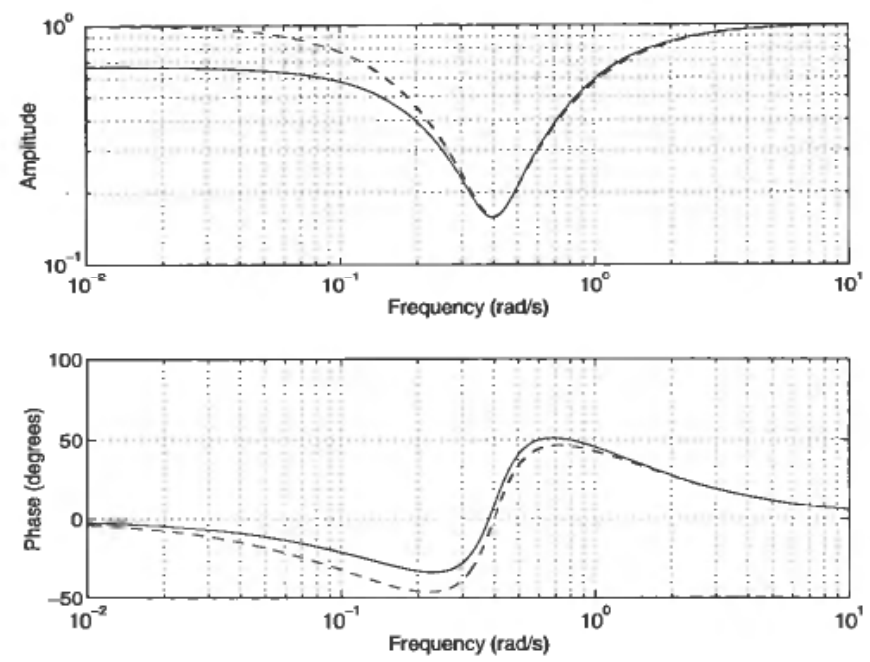

Figure 2. The 'generalized notch filter' $f_{H}(j \omega)$ in (21) (solid lines) and the conventional notch filter $f_{n}(j \omega)$ in (4) (broken lines).

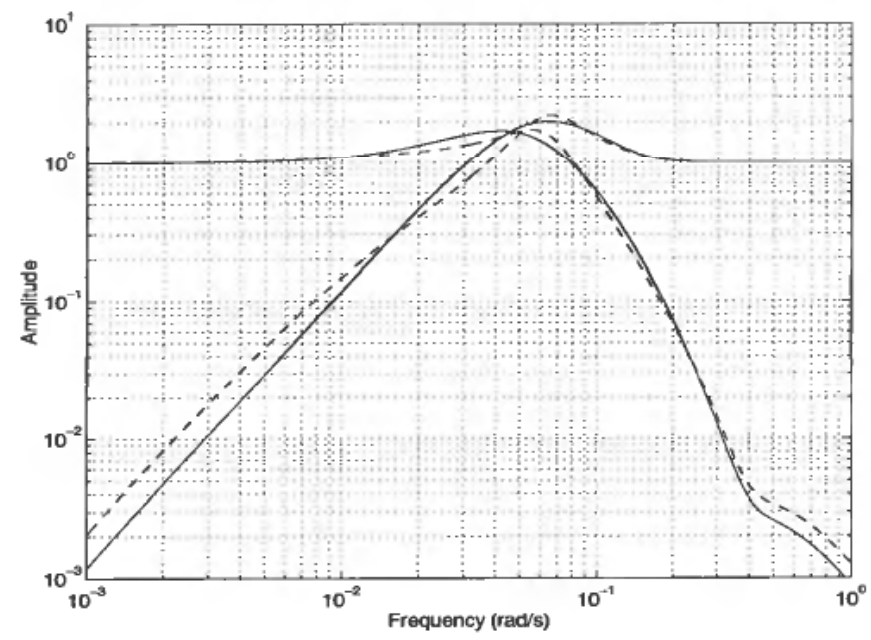

Figure 3. Amplitudes of the sensitivity $(|S(j \omega)|)$ and the complementary sensitivity $(|1-S(j \omega)|)$ for the observer based control system (solid lines) and for the conventional control system (broken lines).

Figure 2 shows the transfer function $f_{n}(s)$ for the notch filter, and the corresponding transfer function $f_{H}(s)$ in (21) for the Kalman filter. The difference between the two filters is the term $k_{4}+2 \zeta \omega_{0} k_{3}$. which is always positive if $\eta_{L}>0$.

The amplitudes of the sensitivity function $S(j \omega)=x(j \omega) / v(j \omega)$, where $x$ is the position of the vessel, and the complementary sensitivity function $1-S(j \omega)$ are shown in Fig. 3. The performances of the two control systems are very similar. The main difference is slightly lower sensitivity at low frequencies for the observer based system.

Thruster modulation for the two control systems are shown in Fig. 4. The notch at $\omega=0.4 / \mathrm{rad} / \mathrm{s}$ is easily recognized for both systems. The noise amplification at frequencies above $0.4 \mathrm{rad} / \mathrm{s}$ is slightly higher with the conventional system. 


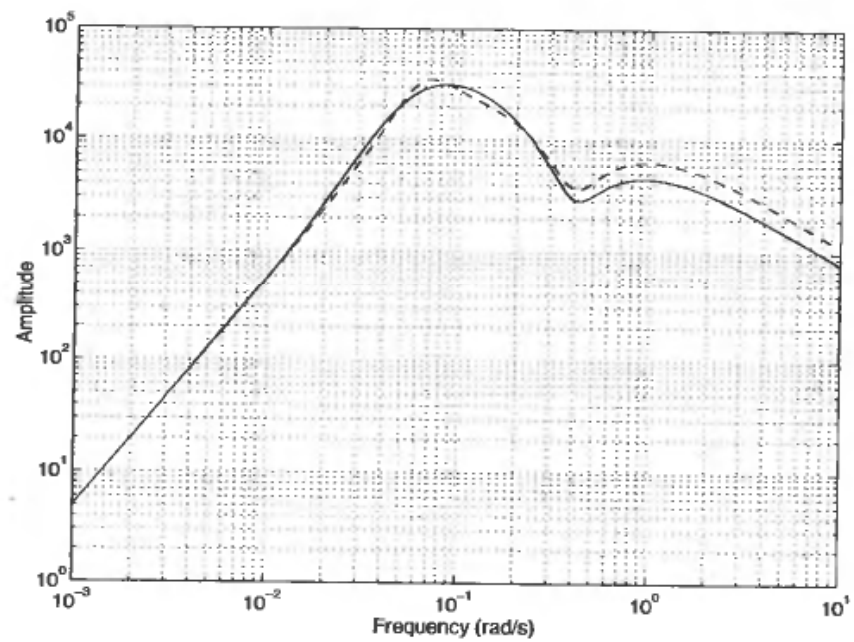

Figure 4. $\left|F_{T}(j \omega) / v(j \omega)\right|$ for the observer based control system (solid line) and the conventional system (broken line).
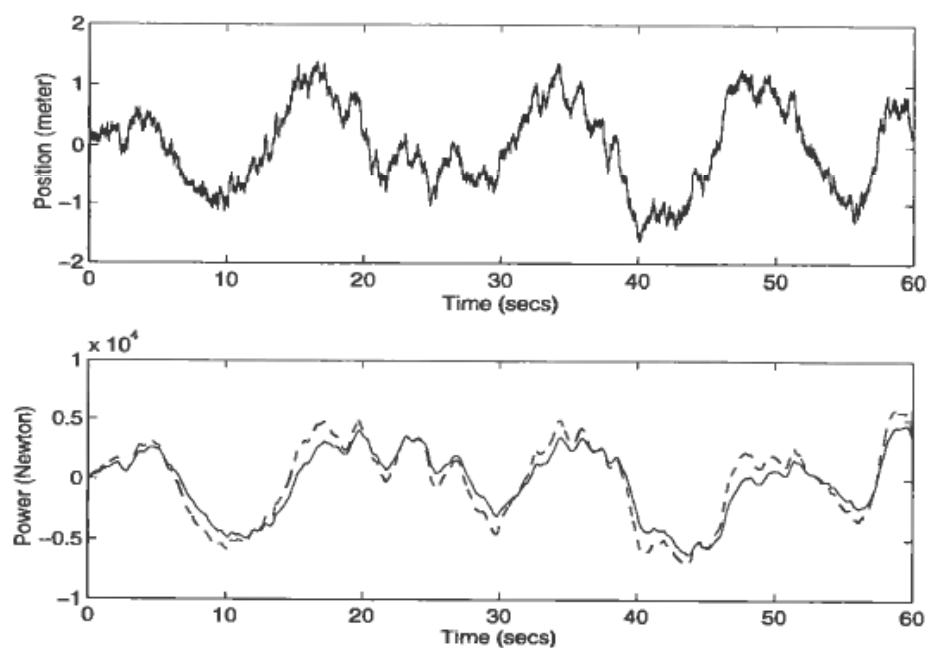

Figure 5. Vessel position (above) and thruster force (below) for the observer based system (solid line) and the conventional system (broken line).

The two systems are now simulated. The disturbances are generated from (2), with the same parameter values as is used for design of the model based filter, however, the low frequency disturbances generated by $\eta_{L}$ in (1) are set to zero. Hence, the only disturbances are the first order wave disturbances. The references for the control systems are set to zero. As can be seen from Fig. 5, the vessel position is almost identical for the two control systems, and the thruster force is also very similar.

\section{Discussion and conclusions}

Three different approaches to wave disturbance filtering in dynamic positioning systems have been studied in this paper. It has been shown that an observer based 
approach leads to approximately the same dynamics in the observer feedback loop as is achieved in the control feedback loop with a conventional notch filter design.

It has also been shown that a passivity based design approach leads to a conventional filter with PD-controller. The filter is very similar to a notch-filter, but there is a relation between the parameters in the filter and the PD-controller, which ensures the passivity properties of the control system. It is interesting to note that this conventional filter has exactly the same structure as the corresponding filter (called 'generalized notch filter') in the observer based approach.

The conclusion from the frequency plots and the simulation is that approximately the same performance can be achieved with the conventional wave filter as with the observer based filter. However, a major advantage with the Kalman filter is that it can be combined with adaptation more easily than a conventional filter. It is an attractive approach to estimate the dominating wave frequency component $\omega_{0}$ (See Fung and Grimble, 1983, Sælid et al. 1983), and recalculate the Kalman filter gains on-line. The gains can also be precalculated and tabulated for varying values of the frequency estimate $\hat{\boldsymbol{\omega}}_{0}$. The optimal filter gains are sensitive for varying values on $\hat{\boldsymbol{\omega}}_{0}$, particularly when $\hat{\omega}_{0}$ is near the bandwidth of the observer loop. Hence, it is essential not to combine a filter with constant filter gains with adaptation on $\omega_{0}$.

\section{REFERENCES}

Balchen, J. G., Jenssen, N. A., Mathisen, E. and Salid, S. (1980). A dynamic positioning system based on Kalman filtering and optimal control. Modeling, Identification and Control, 1, 135-163.

Fossen, T. I. (1994). Guidance and Control of Ocean Vehicles (John Wiley \& Sons, Ltd, Chichester).

Fung, P. T. and GRIMBLE, M. J. (1983). Dynamic ship positioning using a self-tuning Kalman filter. IEEE Trans. Automatic Control, AC-28, 339-350.

Grimble, M. J. and PAtTon, R. J. (1980). The design of dynamic ship positioning control systems using stochastic optimal control theory. Optimal Contr. Applic. \& Methods, 1, 167-202.

JOHANNESEN, E. AA. and EGELAND, O. (1993). Dynamic positioning with wave filtering using a passive controller structure. Proc. IEEE 32nd Conference on Decision and Control, San Antonio, Texas, pp. 1903-1908.

Morgan, M. J. (1978). Dynamic Positioning of Offshore Vessels (Petroleum Publishing Company).

Sargent, J. S. and Cowgill, P. N. (1976). Design considerations for dynamically positioned utility vessels. Offshore Technology Conference, Dallas, U.S.A.

STEIN, G. and ATHANS, M. (1987). The LQG/LTR procedure for multivariable feedback control design. IEEE Trans. Automatic Control, AC-32, 105-114.

SÆlid, S., Jenssen, N. A. and Balchen, J. G. (1983). Design and analysis of a dynamic positioning system based on Kalman filtering and optimal control. IEEE Trans. Automatic Control, AC-28, 331-339. 